\title{
Activité, emploi et chômage en Espagne (1976-2007) : une perspective évolutive et comparée
}

Actividad, empleo y paro en España (1976-2007) : una perspectiva evolutiva y comparada

Activity, employment and unemployment in Spain, in a compared and gradual perspective (1976-2007)

\section{Luis Toharia}

\section{CpenEdition}

\section{Journals}

Édition électronique

URL : http://journals.openedition.org/travailemploi/4189

DOI : 10.4000/travailemploi.4189

ISSN : $1775-416 \mathrm{X}$

\section{Éditeur}

DARES - Ministère du Travail

Édition imprimée

Date de publication : 15 septembre 2008

Pagination : 13-27

ISSN : 0224-4365

\section{Référence électronique}

Luis Toharia, «Activité, emploi et chômage en Espagne (1976-2007) : une perspective évolutive et comparée », Travail et Emploi [En ligne], 115 | juillet-septembre 2008, mis en ligne le 18 février 2011 , consulté le 23 avril 2019. URL : http://journals.openedition.org/travailemploi/4189; DOI : 10.4000/ travailemploi.4189 


\title{
Activité, emploi et chômage en Espagne (1976-2007): une perspective évolutive et comparée
}

\author{
Luis Toharia (*)
}

L'objet de cet article est de présenter de façon synthétique les traits les plus saillants et les plus représentatifs du marché du travail espagnol. Nous montrerons dans une première partie son évolution à partir de 1976 - année du début de la transition de la société espagnole vers un ordre social démocratique: nous aborderons cette question d'abord sous l'angle des paramètres les plus classiques et les plus habituellement utilisés par les experts en économie du travail (activité, occupation et chômage), puis sous l'angle de l'évolution du comportement «économique» des hommes et de femmes, dimension qui est à l'origine d'un changement qualitatif profond. Une seconde partie abordera le chômage comme indicateur de la diversité de la population active et de son comportement sur le plan social et économique. Dans un troisième temps, nous mettrons en évidence les aspects propres au marché $\mathrm{du}$ travail espagnol par rapport à d'autres pays européens et à l'Europe des quinze. Cette comparaison nous amènera à mettre en exergue dans un quatrième temps certaines carences du marché de l'emploi en Espagne.

\section{Évolution générale de l'emploi et du chômage (1976-2007)}

Comme nous l'avons indiqué, nous aborderons dans un premier temps les aspects les plus généraux de l'évolution du marché du travail espagnol, puis les aspects relevant de l'évolution différente du marché du travail pour les hommes et pour les femmes. Nous laisserons de côté un point clé pour la compréhension de cette question, celui de l'ana-

(*) Universitad de Alcalà, Departemento. de Fundamentos de Economía e Historia Económica, luis.toharia@uah.es. L'auteur voudrait remercier le comité de rédaction de la revue, ainsi que Carlos Prieto et Inmaculada Cebrán, dont les commentaires à une première version de l'article ont été extrêmement utiles pour rédiger la version finale. Les erreurs et lacunes qui peuvent subsister sont de la responsabilité exclusive de l'auteur. lyse en profondeur du rapport entre l'évolution de l'emploi, de la croissance et de la productivité, point qui sera traité dans l'article de Inmaculada Cebrián dans ce numéro.

\section{Activité, emploi et chômage (1976-2007): aspects généraux}

Pour bien comprendre la situation actuelle du marché du travail en Espagne, il est nécessaire d'apprécier l'évolution de l'activité, de l'emploi et du chômage pendant une période suffisamment longue pour pouvoir observer des régularités significatives(1). Notre analyse démarre en 1976. Il s'agit d'une date remarquable du point de vue aussi bien économique que social et politique (et même statistique). Il s'agit de l'année où commence la transition politique vers un ordre social démocratique qui va affecter profondément tous les aspects de la société espagnole. Concernant les informations statistiques sur l'emploi, c'est aussi la première année à partir de laquelle des données cohérentes ont été reconstruites. Le graphique 1 présente l'évolution de la population active, de l'emploi et du chômage au cours des trente dernières années.

En 1976, l'Espagne se trouve à un moment difficile du point de vue économique, social et politique. $\mathrm{Au}$ cours des années précédentes, l'emploi s'était accru à un taux modéré mais suffisant pour suivre le rythme de la population active, dont la croissance était limitée par l'émigration, notamment vers les autres pays européens, ce qui entraînait des niveaux de chômage pratiquement nuls. La mort du dictateur Franco en 1975 avait marqué le début d'une période pleine d'incertitudes sur le plan politique qui, en pleine crise pétrolière mondiale, se traduisait en une stagnation de la population active et de l'emploi, mais aussi par une inflation de plus de $25 \%$ par an. Les «Pactes de la Moncloa» signés fin

(1) La première partie de cette section est basée sur TOHARIA (1998). La comparaison des deux textes montre à quel point la situation de l'économie et du marché de travail a changé en Espagne au cours des dix dernières années. On pourra consulter aussi Toharia, Malo (2000) et Toharia (2005). 
Graphique 1 : Population active, emploi et taux de chômage en Espagne (1976-2007)

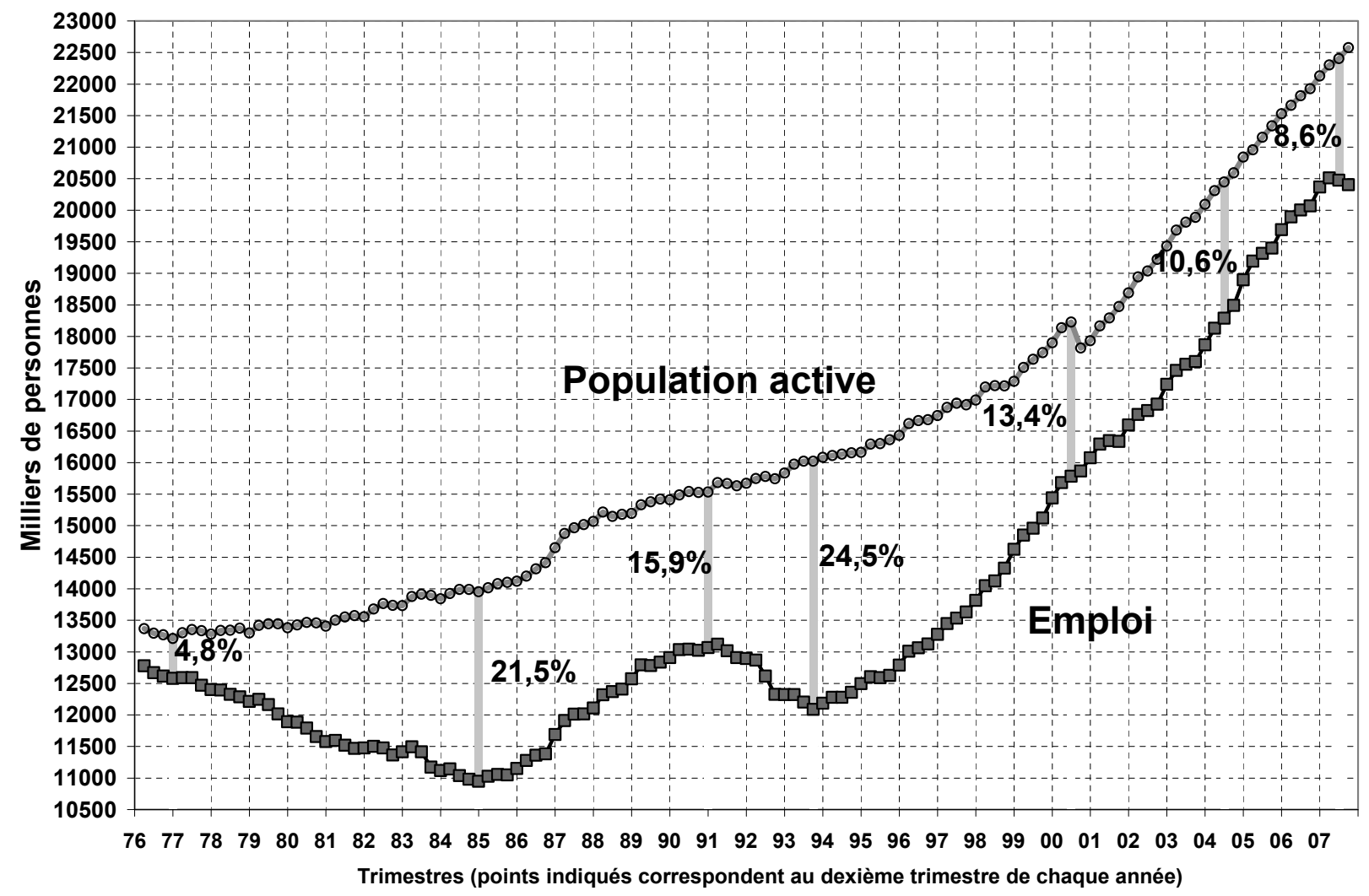

Source: enquête sur la population active (EPA), pondérations recalculées en 2005.

Note de lecture: le graphique présente les séries trimestrielles de la population active et de l'emploi depuis 1976; le chômage est indirectement représenté par la différence entre les deux séries, et aussi plus directement par les colonnes de couleur gris clair qui le quantifient à divers moments du temps, en indiquant le taux de chômage en pourcentage de la population active. Sur la chute de la population active observée en 2001, voir l'encadré 1 sur les méthodes de mesure du chômage en Espagne.

1977, quelques mois après les premières élections générales de la nouvelle période démocratique, marquèrent le début de ce qui pourrait être considéré comme la transition économique.

Quatre sous-périodes peuvent être clairement distinguées au long des trois décennies qui suivirent (voir graphique 1):

A) La crise de l'emploi, 1977-1985: pendant cette période, l'économie espagnole subit d'importantes pertes d'emploi, liées à différents facteurs : la seconde crise du pétrole; le rattrapage technologique et l'ajustement du système productif (trop longtemps dominé par les conditions protectionnistes du régime franquiste, et par l'absence d'un système libre de relations professionnelles); les nouvelles conditions de concurrence requises par l'entrée imminente dans la Communauté européenne; enfin, une politique macro-économique de réduction de l'inflation, qui comporte une politique monétaire restrictive et une négociation à la baisse des salaires nominaux avec les centrales syndicales. La réduction de l'emploi se traduit par une hausse jusque-là inconnue du chômage. Cette hausse demeure cependant atténuée par la croissance relativement faible de la population active, surtout avant 1981. À partir de 1981, les cohortes nées dans les années 1960, légèrement plus nombreuses que les précédentes mais surtout bénéficiant de meilleures conditions sanitaires et, par conséquent, d'une plus basse mortalité infantile, arrivent sur le marché du travail. Au point le plus bas de la crise, en 1985, le taux de chômage atteint $21,5 \%$ de la population active. Il faut noter que c'est à ce moment-là que les premières critiques sur les méthodes de mesure du chômage se font entendre.

B) L'expansion de la seconde moitié des années 1980: à partir du deuxième trimestre 1985, l'économie espagnole retrouve le chemin de la croissance économique et de la création d'emploi. Entre 1985 et 1991, l'emploi s'accroît à des taux sans précédent dans l'histoire espagnole. Plusieurs facteurs peuvent être mentionnés pour expliquer ce tournant de la conjoncture et la vigueur de la croissance. D'une part, la situation cyclique mondiale, en expansion depuis 1983, finit par toucher l'économie espagnole, ce que renforce la forte chute des prix du pétrole en 1985; l'accession à la Communauté européenne en 1986 fournit une impulsion additionnelle. D'autre part, la bonne situation des profits des entreprises, comme conséquence de la période d' ajustement de la première moitié des années 1980, où les salaires réels avaient notablement reculé, alimente les processus d'investissement. Enfin, la réforme du marché du travail 
introduite en 1984 facilite l'utilisation sans restriction des contrats à durée déterminée, y compris pour des tâches normales c'est-à-dire non-temporaires; cette réforme ajoute un élément particulier à la croissance de l'emploi, en introduisant une segmentation entre les travailleurs anciens, fortement protégés contre les licenciements, même justifiés par des raisons économiques, et les «nouveaux», qui sont pour la plupart sur des contrats de six mois à la fin desquels l'employeur peut choisir entre la fin de la relation de travail ou le renouvellement pour une autre période de six mois (jusqu'à un maximum de trois ans)(2). Cependant du fait de l'accroissement important de la population active, notamment féminine, la forte expansion de l'emploi (1,8 million en cinq ans, soit un taux moyen de croissance de $3 \%$ par an) ne se traduit que par une réduction très modérée des niveaux de chômage (soit une réduction de 500000 personnes). Cet accroissement de la population active féminine est seulement pour partie la réaction à la bonne situation de l'emploi et ce pour deux raisons: d'une part, l'activité féminine commence à augmenter en 1983, c'està-dire, avant la reprise économique de la deuxième partie des années 1980; d'autre part, la croissance est persistante jusqu'à nos jours, aussi bien dans les périodes d'expansion que dans celles de récession. Il s'agit plutôt d'un changement de tendance fondamental dans le comportement des femmes vis-à-vis du marché de travail (et de la fécondité). En tout cas, bien que le taux de chômage atteigne encore $16 \%$ en 1991, certains secteurs de l'économie souffrent néanmoins de pénuries de qualifications, ce qui s'explique par la nature du chômage, très biaisé envers les jeunes et les femmes (le taux de chômage des «personnes de référence» des ménages se situe en dessous de $7 \%$ à cette époque).

C) La crise de 1992 à 1994 : entre 1991 et 1994, l'économie espagnole subit une très grave récession qui entraîne des pertes d'emploi plus intenses que celles enregistrées au cours de la crise précédente. Parmi les causes sous-jacentes à la crise, on peut mentionner la crise internationale qui, cette fois-ci, frappe synchroniquement l'économie espagnole, la perte de compétitivité des produits espagnols comme conséquence du haut taux de change de la peseta ainsi que la croissance des déficits budgétaires, la fin du cycle expansionniste lié aux évènements de 1992 (les Jeux olympiques de Barcelone, l'Exposition universelle de Séville), les hausses de salaires résultant de la grève générale de 1988 et aussi la limitation introduite en 1992 à l'utilisation des contrats à durée déterminée, bien que l'effet de cette dernière mesure ne soit pas très souvent mentionné, probablement à cause des difficultés à l'évaluer proprement. Une des caractéristiques les plus intéressantes de cette crise est qu'elle ne se centre pas seulement

(2) Sur le modèle espagnol établi par la réforme de 1984, on pourra consulter ToHARIA, MALO (2000). sur les travailleurs employés à durée déterminée, comme les experts l'avaient annoncé, mais qu'elle affecte d'une manière très significative les salariés ayant des contrats à durée indéterminée: en 1993, les licenciements indemnisés représentent près de $5 \%$ des salariés du secteur privé, pour une moyenne de $3 \%$ les années précédentes. Comme en 1985, la forte crise du chômage déclenche une seconde vague de critiques des instruments de mesure, notamment de l'enquête sur la population active (EPA) (voir encadré 1 sur la mesure du chômage et de l'emploi en Espagne).

D) La reprise récente, de 1994 à 2007: depuis 1994, l'économie espagnole est entrée dans une période fortement expansionniste qui se poursuit encore treize ans plus tard, à la surprise de tous les experts, mais qui semble être sur le point de retomber au début 2008. Il s'agit d'une période extrêmement longue de croissance persistante, facilitée au début par les réformes économiques, la réforme de la monnaie et celle du marché du travail introduites en 1993, puis plus tard par l'expansion générale de l'économie européenne, renforcée par la troisième phase de l'Union économique et monétaire et l'arrivée de l'euro, ainsi que par le «choc» positif de l'offre de travail représenté par l'immigration. Au total, l'emploi s'est accru entre 1994 et 2007 de plus de 8 millions de personnes, soit $67 \%$. Les presque 4 millions de chômeurs comptabilisés en 1994 ont été largement insuffisants pour faire face à un tel accroissement, surtout si l'on considère qu'ils sont encore 1,8 million en 2007. Il est évident, par conséquent, que cet accroissement a été possible grâce à l'augmentation de la population active. Deux facteurs sont importants à cet égard: la croissance persistante, depuis 1983, de l'activité féminine autochtone (voir l'article de Teresa Torns plus loin) et, surtout, l'entrée massive, notamment à partir de la deuxième moitié des années 1990, de personnes provenant d'autres pays, tels que le Maroc et certains pays sud-américains, principalement l'Equateur, la Bolivie et la Colombie, mais aussi les pays de l'Est de l'Europe (la Roumanie devenant récemment le principal fournisseur de main-d'œuvre). Les enjeux posés par ces flux d'immigrants, auxquels la société espagnole doit répondre, seront analysés plus en détail dans l'article de Lorenzo Cachón. Il est également vrai que la réduction du chômage a été en partie artificielle, du fait de l'application du nouveau règlement adopté par la Commission européenne en 2000 qui, d'après divers observateurs, visait le taux de chômage «trop élevé» de l'Espagne du fait d'une prise en compte insuffisante du travail au noir (voir encadré 1 infra). La réduction postérieure à des changements introduits en 2005 peut elle aussi être attribuée, partiellement du moins, à ces ajustements, mais cela est beaucoup moins clair (et aussi moins étudié). En tout cas, les chiffres de l'emploi sont beaucoup moins discutables, car ils sont plus en phase avec les données 
Graphique 2: Population active, emploi et taux de chômage en Espagne, par sexe, (1976-2007)

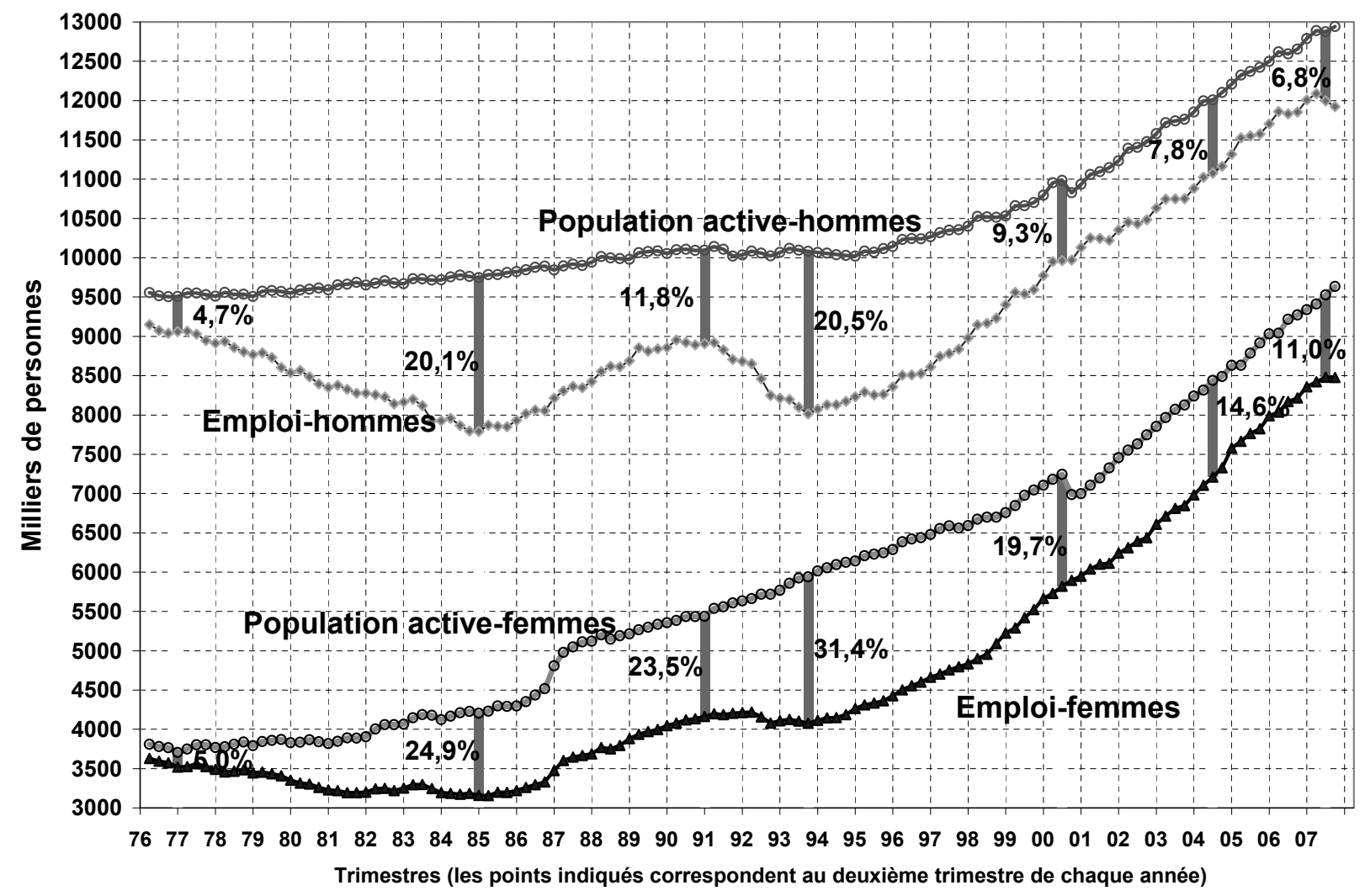

Source: enquête sur la population active (EPA), pondérations recalculées en 2005.

Note de lecture: le graphique présente les séries trimestrielles de la population active et de l'emploi depuis 1976; il s'agit d'un graphique semblable au graphique 1 mais les chiffres se présentent séparément pour hommes et femmes. Il faut noter que l'échelles est la même, ce qui met en exergue les grandes et persistantes différences entre les deux sexes. Sur la chute de la population active observée en 2001, voir l'encadré 1 sur les méthodes de mesure du chômage en Espagne.

administratives de la Sécurité sociale. Quoi qu'il en soit, il est généralement accepté en Espagne que les divers changements introduits dans l'EPA, même s'ils sont discutables d'un point de vue strictement méthodologique (comme il arrive dans tous les pays à économie avancée), ont rapproché les chiffres de l'emploi et du chômage de la perception qu'en avaient les travailleurs espagnols.

En somme, l'économie espagnole a subi, au cours des trente dernières années, deux phases de forte destruction de l'emploi et deux autres de forte croissance. La grande crise de la première période et l'énorme reprise des dernières années sont particulièrement remarquables. L'année 2008 s'annonce peut-être comme le début de la troisième crise, liée à la chute maintes fois annoncée - et semblet-il finalement arrivée -, du secteur du bâtiment. Il reste à savoir en effet si l'économie espagnole a été capable de fabriquer un tissu économique plus solide et si les ombres d'une croissance dépendant du secteur de la construction dissimulent les fondements plus stables des secteurs de l'industrie et des services productifs.

\section{Des différences entre les hommes et des femmes}

L'évolution qui vient d'être présentée doit être nuancée lorsque les données sont analysées séparément pour les hommes et pour les femmes (3). Le graphique 2 présente l'information qui était contenue dans le graphique 1 désagrégée par sexe.

En ce qui concerne les hommes, l'emploi suit un parcours qui ressemble beaucoup à celui de l'ensemble de l'économie, bien qu'avec des oscillations plus prononcées et symétriques, de telle sorte que la crise des années 1990 porte l'emploi à un niveau presque égal à celui de 1985, balayant ainsi en trois années la forte croissance des six années précédentes, mais laissant aussi une structure certainement différente. Les deux reprises arrivent à rejoindre le niveau initial, mais la seconde le surpasse largement, de telle sorte que le niveau d'emploi masculin en 2007 est supérieur d'environ

(3) Sur les différences entre les femmes et les hommes sur le marché du travail espagnol, voir CEBrián et Moreno (2007); la situation comparée du point de vue des ménages et du cours de la vie est analysée dans Cebrián, Moreno et ToHaria (2007) et aussi dans AnXo, Cebrián, Fagan et Moreno (2007). 


\section{Encadré 1 \\ La mesure du chômage et de l'emploi en Espagne}

Comme dans la plupart des pays européens, il y a en Espagne deux sources statistiques qui sont utilisées pour mesurer le nombre de chômeurs à un moment donné. D'une part, l'Institut national de la statistique espagnol (Instituto Nacional de Estadística) (INE), réalise depuis 1964 une enquête sur la population active (EPA), de périodicité trimestrielle depuis 1976, qui s'accorde aux critères d'Eurostat, les résultats du deuxième trimestre de chaque année servant de base pour la section espagnole de l'enquête européenne sur les forces de travail. D'autre part, le Service public de l'emploi de l'État (SPEE), agence qui dépend du ministère du Travail, publie mensuellement le nombre de chômeurs enregistrés par les services publics de l'emploi, sur la base d'un décret gouvernemental de 1985 qui définit les critères à suivre pour considérer comme chômeurs ou non les demandeurs d'emploi qui s'inscrivent comme tels dans les dits services.

L'EPA fut l'objet de deux grandes vagues de critiques dans les années 1980 et 1990, en coïncidence avec les valeurs les plus élevées du taux de chômage. Le contenu de ces critiques portait principalement sur le fait que l'EPA ne prenait pas correctement en compte dans ses mesures l'emploi «au noir ", considéré comme très significatif en Espagne et comme le facteur explicatif de la paix sociale régnant dans un pays frappé par un chômage très élevé d'après l'EPA, mais qui dans les faits, selon ces critiques, ne l'était pas tant que cela. En fait, ces critiques n'étaient pas vraiment fondées, car l'EPA a toujours suivi de près les recommandations d'Eurostat, et l'appréciation des experts en la matière a été, dans tous les cas, claire: l'emploi au noir existait certainement en Espagne, mais l'EPA, qui est une enquête auprès des ménages, était capable d'en capturer une proportion significative. D'autre part, le poids du chômage retombait sur les familles qui pouvaient le supporter beaucoup mieux qu'on ne pouvait le penser à partir de chiffres du chômage individuel.

Néanmoins, il devenait de plus en plus évident, spécialement au vu de l'évolution des registres de la Sécurité sociale, que les chiffres de l'emploi de l'EPA (et pas tellement ceux du chômage) sous-estimaient la vraie grandeur de la variable de l'emploi. Cela donna lieu à quatre réformes successives de l'EPA, qui ont combiné des changements dans les procédures de l'enquête et l'utilisation de nouvelles estimations de la population totale qui servent de base à ses estimations. Les deux dernières réformes sont certainement les plus significatives.

En 1995, les chiffres de la population furent ajustés aux résultats du recensement de 1991.

En 1999, le questionnaire fut adapté aux nouvelles recommandations d'Eurostat pour homogénéiser les enquêtes au niveau européen, ce qui impliqua, dans le cas de l'EPA espagnole, des ajustements très limités, puisqu'elle suivait déjà les recommandations précédentes. Néanmoins, les changements eurent un effet de réduction du chômage, à cause de la nouvelle rédaction de la question de base relative à la recherche d'un emploi.

En 2002, un triple changement fut introduit. En premier lieu, les populations furent ajustées pour prendre en compte les entrées d'immigrants qui avaient été perçues dans les premiers résultats du recensement de 2001; en deuxième lieu, les facteurs de pondération furent recalculés de telle sorte que la structure par âge et sexe de la population coïncide avec les estimations provenant des chiffres du recensement, dont le décalage représentait un problème longuement dénoncé par les experts et était l'un des principaux facteurs de sous-estimation de l'emploi; finalement, le nouveau règlement 1897/2000 de la Commission européenne fut appliqué en ce qui concerne la définition du chômage. L'effet combiné de ces changements emporta une forte augmentation des chiffres de l'emploi et une réduction du chômage estimée à environ $20 \%$, du fait de l'application du nouveau règlement ${ }^{(1)}$. L'effet du nouveau règlement est très clairement visible dans le graphique 1 : la chute observée au premier trimestre 2001 (premier trimestre auquel le nouveau règlement est appliqué) dans la courbe qui représente la population active n'est pas observée dans celle de l'emploi, ce qu'indique qu'elle relève de la réduction du chômage. La diminution du taux de chômage de 13,4\% au quatrième trimestre 2000 à $10,6 \%$ quatre ans après, est par conséquent en grande partie une construction statistique: en effet, au premier trimestre de 2001, le taux était déjà de 10,9\%.

Finalement, en 2005, deux changements furent introduits. D'une part, toute la série historique depuis 1996 fut recalculée à partir des nouvelles projections de la population fondées non seulement sur le recensement de 2001 mais aussi, et surtout, sur le registre municipal de la population, devenu la meilleure source pour connaître le vrai chiffre de la population étrangère, même en situation irrégulière. D'autre part, des adaptations méthodologiques furent introduites dans l'enquête, aussi bien dans le questionnaire que dans les méthodes de recueil de l'information, qui désormais seraient principalement basées sur un système d'interviews téléphoniques assistés par ordinateur (CATI). Le nouveau système se révéla être un peu instable dans les premiers trimestres de mise en place, mais il semble avoir acquis une certaine maturité après deux ans de fonctionnement. Le problème de l'estimation du «vrai » chiffre de la population demeure, cependant, à cause des problèmes posés par le registre de la population qui pourrait surestimer la population étrangère. II n'est pas impossible, par conséquent, bien qu'assez peu probable, que de nouvelles réformes, ayant pour effet de modifier les chiffres cette fois-ci légèrement à la baisse, ne se produisent dans les prochaines années.

Quant aux statistiques sur les «chômeurs enregistrés", la réforme introduite en 2005 dans le cadre de la décentralisation territoriale des services publics de l'emploi a fait ressortir les problèmes en ce qui concerne les chiffres publiés depuis de nombreuses années, du fait d'une application trop généreuse des critères d'exclusion des inscrits. Les nouveaux chiffres sont maintenant au-dessus de ceux de l'EPA. 
Depuis les dernières réformes, les chiffres de l'EPA sont en général considérés comme valables et ceux du chômage enregistré demeurent comme un complément statistique dont l'avantage est qu'il est publié tous les mois et qu'il permet une analyse plus détaillée du point de vue territorial. Les séries actuellement existantes peuvent être considérées comme comparables, à la seule exception de la rupture de 2001. L'INE a publié une série révisée des chiffres agrégés du chômage qui corrige vers le passé le décalage existant. Cependant les chiffres de cette étude ne sont pas considérés comme officiels et la rupture entre les deux sources persiste, bien que de moins en moins importante au fur et à mesure que le temps avance.

(1) Pour une analyse plus détaillée des effets du nouveau règlement, on pourra consulter GARRIDO et ToHARIA (2004).

un tiers à celui observé trente ans auparavant, soit un accroissement net de presque 3 millions d'emplois. Cette évolution de l'emploi entraîne derrière elle les niveaux de chômage qui, par deux fois, arrivent à surpasser $20 \%$ de la population active, mais qui descendent assez rapidement, pour chuter jusqu'à moins de $12 \%$ en 1991 et, ce qui est plus impressionnant, à 6,2\% en 2007, valeur très proche du chômage observé en 1976. Cette évolution du chômage est rendue possible jusqu'en 1996 par le développement relativement lent de la population active masculine. Cette population augmente d'un peu plus de 500000 personnes entre 1976 et 1996, mais elle accélère son rythme de croissance à partir de cette date, du fait de l'entrée d'immigrants qui rend possible l'augmentation et de l'emploi et de la population active. Entre 1996 et 2007, la population active masculine augmente de près de 3 millions de personnes, soit environ $27 \%$.

Quant aux femmes, leurs niveaux demeurent tout au long de la période de trente ans étudiée audessous de ceux des hommes, mais leurs tendances sont beaucoup plus ascendantes, aussi bien en ce qui concerne l'emploi qu'en ce qui concerne la population active. En effet, les crises de l'emploi affectent la population féminine de façon beaucoup plus atténuée, ce qui est particulièrement vrai lors de la crise des années 1990: ceci s'explique par la nature même de cette crise, qui porta surtout sur les secteurs industriels et du bâtiment, les moins fournisseurs d'emploi pour les femmes. On peut dire que, sauf le léger répit de 1992-1994, l'emploi féminin n'a cessé de s'accroître depuis 1985, pour une augmentation totale proche de 5,5 millions de personnes, soit plus du double et presque le triple du niveau initial. Si l'on considère la période totale de trente ans couverte par le graphique, la part des femmes dans l'emploi total passe de $28 \%$ à plus de $41 \%$.

En ce qui concerne la population active, la tendance est également croissante et quasiment équivalente à celle de l'emploi, et cela depuis le début des années 1980. Deux bonds d'origine méthodologique peuvent être clairement perçus dans la série, le premier vers le haut, lors de l'introduction de la nouvelle enquête sur la population active (EPA) en 1987 (deuxième trimestre), le second en sens inverse, lors de l'application en 2001 du nouveau règlement de la Commission européenne, qui affecte davantage les femmes que les hommes (voir encadré 1 supra). En tout cas, la participation croissante des femmes au marché du travail est évidente et provient principalement de la modernisation de la conduite des jeunes femmes: elles ne suivent plus le modèle traditionnel qui consistait à travailler quand elles sont jeunes, à abandonner l'emploi pour avoir des enfants et à y retourner plus tard. Les Espagnoles entrent sur le marché du travail et ne l'abandonnent que de façon très partielle. La conséquence en est un délai pour avoir un premier enfant et une baisse très notable des taux de fécondité.

L'évolution constante à la hausse de la population active féminine empêche que la bonne conduite de l'emploi se traduise par une réduction plus prononcée du chômage. Alors que la première crise porte le chômage masculin à des niveaux supérieurs à $20 \%$, dans le cas des femmes, l'apparition du chômage s'explique pour moitié par la perte d'emplois et pour moitié par l'accroissement de la population active. La mauvaise situation de l'emploi ne freine pas la participation des femmes au marché du travail: elles y entrent pour y rester, même si les conditions d'emploi ne sont pas les meilleures. Au cours des années suivantes, la course de l'emploi en quête de population active produit lentement les effets attendus, de sorte qu'en 2006, le taux de chômage féminin est proche finalement du seuil des $10 \%$, bien que demeurant à un niveau qui est presque le double de celui des hommes.

En somme, le marché du travail féminin se caractérise par une augmentation continue de la population active, qui doit être mise en relation avec le changement d'attitude des femmes appartenant aux cohortes relativement jeunes (4) qui abandonnent le cours traditionnel de retrait du marché du travail (si jamais elles y étaient entrées jeunes) lors du mariage, pour prendre soin des enfants, conformément à un modèle plus «masculin » où le travail payant prime sur les tâches familiales: ceci a entraîné un retard de la natalité qui a amené les taux de fécondité espagnols jusqu'aux niveaux les plus bas du monde, tendance inversée seulement dans les dernières années grâce à un taux de fécondité

(4) D'après GARRIDo (1993), le point de rupture concerne les femmes nées entre 1950 et 1955 . 
Graphique 3: Taux d'emploi de la population des 16-64 ans en Espagne par sexe (1976-2007)

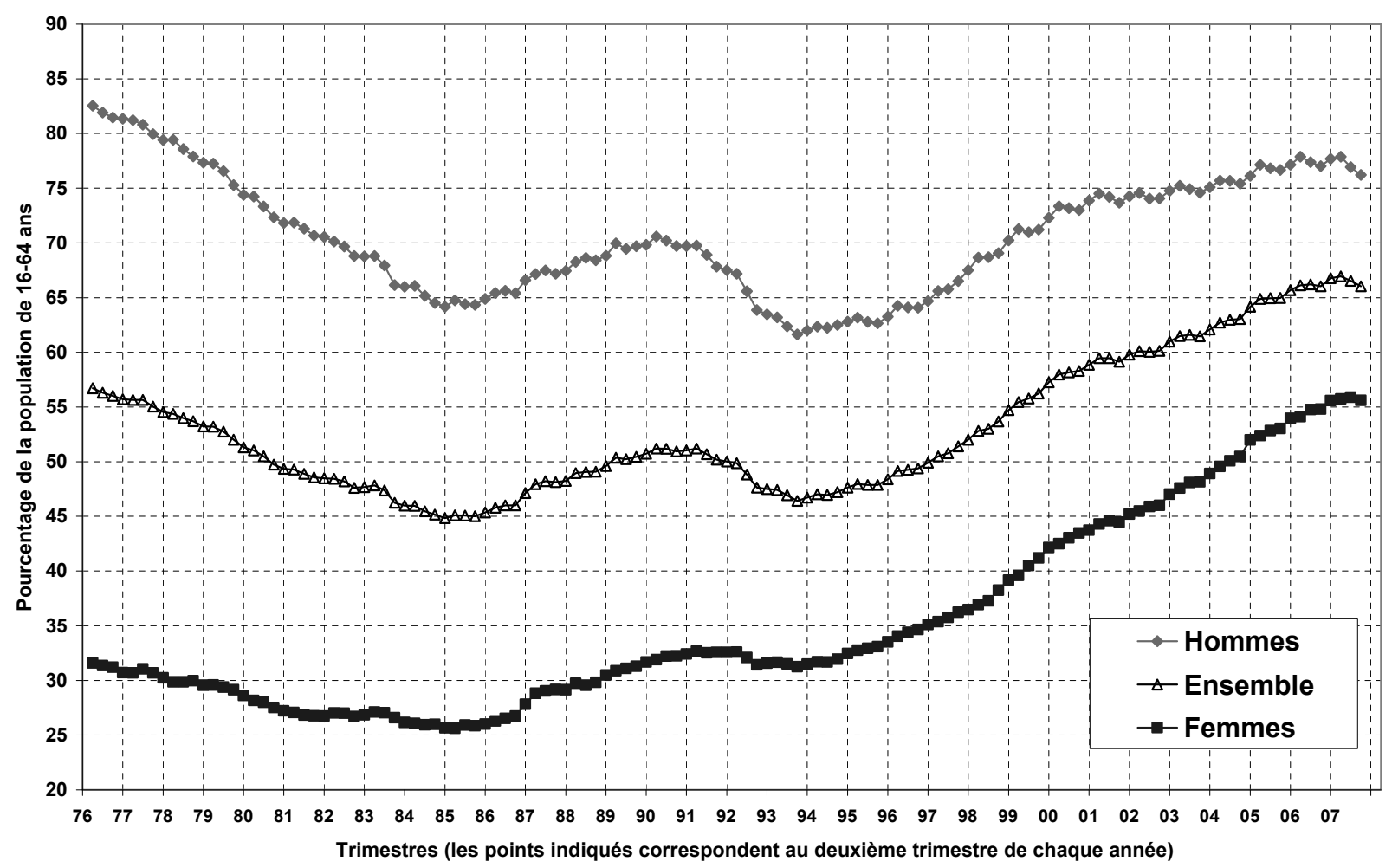

Source: enquête sur la population active (EPA), pondérations de 2005

Note de lecture: le graphique montre les données trimestrielles du taux d'emploi, qui mesure le rapport emploi-population de 16-64 ans. Pour les hommes, le taux suit une évolution en forme de $\mathrm{W}$ horizontale alors que pour les femmes, la $\mathrm{W}$ est beaucoup plus ouverte et penchée vers la gauche. La distance entre le taux des femmes et celui des hommes passe de 50 points de pourcentage a 20 environ au cours des trente années couvertes par le graphique.

supérieur parmi les immigrantes (voir l'article de Teresa Torns).

Pour résumer l'évolution de l'emploi au cours des trente dernières années, le graphique 3 présente le taux d'emploi (pourcentage de l'emploi par rapport à la population de 16 à 64 ans), qui est l'indicateur retenu par l'Union européenne lors des sommets de Lisbonne (2000) et Stockholm (2001) pour le monitoring des marchés du travail européens (avec la seule exception que la définition de ces sommets faisait référence à la population des 15-64 ans au lieu des 16-64 ans; ce qui implique que les objectifs relatifs à cette population plus réduite devraient être plus élevés d' 1 ou 2 points). Le taux pour l'ensemble de la population, qui était au début de la période de $56,7 \%$, parvient à descendre par deux fois, en 1985 et en 1994, à des niveaux d'environ $45 \%$, mais la reprise des douze dernières années a repoussé cet indicateur vers son niveau historiquement le plus élevé; à $66,1 \%$ au troisième trimestre 2006, il donne à penser que l'Espagne est finalement en mesure de réussir à atteindre les objectifs fixés par les sommets européens, ce qui était simplement impensable à la fin du $\mathrm{XX}^{\mathrm{e}}$ siècle.

L'évolution du taux d'emploi pour les hommes révèle un comportement assez symétrique, de telle sorte que $80 \%$ des hommes entre 16-64 ans ont actuellement un emploi, le même chiffre observé trente ans auparavant. Dans le cas des femmes, étant donné que le niveau de départ était tellement bas, l'accroissement est encore plus spectaculaire: le taux d'emploi arrive presque à doubler, passant de 31,6\% en 1976 à 54,1\% en 2006, chiffre qui, en tout cas, est le double de celui qui s'observe au point le plus bas de la série (25,6\% en 1985), et qui lui aussi suggère que l'Espagne pourrait atteindre l'objectif de $60 \%$ de taux d'emploi pour les femmes en 2010.

Naturellement, il se pourrait que l'économie espagnole craque et que l'emploi s'effondre, comme il arriva au début des années 1990, et comme les données les plus récentes, déjà recueillies dans le graphique 3 , le suggèrent. Les enjeux auxquels elle devra faire face seront analysés dans l'article de Cebrián. 


\section{Le chômage comme indicateur de la diversité de la composition et du comportement de la population active}

Bien que le taux de chômage actuel (2007) en Espagne soit le plus bas des vingt-cinq dernières années, il demeure à un niveau supérieur aux $8 \%$ de la population active (5). Le chômage est un phénomène socio-économique qui a un sens en lui-même. Néanmoins, du point de vue analytique, nous allons nous en servir comme d'une variable instrumentale qui peut rendre visible la diversité de la composition sociale de la population active espagnole et surtout de son comportement différencié par rapport à l'emploi. Nous le ferons en considérant que le chômage est beaucoup plus qu'une pure mesure statistique; comme phénomène socio-économique, il est aussi l'expression des différences de comportement des entreprises vis-à-vis de la diversité des demandeurs d'emploi et de la diversité d'attitude des demandeurs vis-à-vis de l'emploi offert. Ainsi on analysera dans cette section l'incidence différente du chômage en 1996 et en 2007, selon diverses dimensions telles que l'âge, la nationalité et le niveau d'éducation. Toutes les analyses sont présentées désagrégées selon le sexe. Les données font référence à des valeurs moyennes de l'année.

Le tableau 1 contient des informations relatives aux taux de chômage selon les diverses variables mentionnées. Le premier point à mentionner est que la population étrangère représente déjà un pourcentage significatif des chômeurs. Alors qu'en 1996, à peine $1 \%$ des chômeurs étaient des citoyens de pays hors Union européenne (Union européenne à quinze), en 2007 le chiffre dépasse 15\% (pour une population étrangère qui ne représente que $11,4 \%$ de la population active totale). Ce chiffre, équivalent à 300000 personnes, implique que le taux de chômage des «Espagnols»(6), est de 8,1\%, c'est-à-dire, un demi-point de pourcentage en dessous du taux de la population totale, soit $8,6 \%$ (le chiffre pour la population étrangère hors Union européenne étant de $12 \%$ ). C'est pour cette raison que, pour compléter les informations contenues dans les tableaux 1 et 2, trois colonnes ont été ajoutées qui présentent les chiffres correspondant à la population espagnole (y compris les citoyens des autres pays de l'Union européenne).

Si l'on considère tout d'abord les différences par tranches d'âge, la réduction des niveaux de chômage affecte tous les âges, mais plus particulièrement les jeunes et les jeunes adultes. En effet, si en 1996, le chômage décroissait avec l'âge et devenait plus ou

(5) Le dernier chiffre publié, correspondant au premier trimestre de 2008 , le situe déjà à $9,6 \%$.

(6) Y compris ceux qui sont nés hors de l'Espagne, ceux qui ont une double nationalité et les citoyens des autres pays de l'Union européenne à quinze. moins stable à partir de 35 ans chez les hommes (à environ $12 \%$ ), mais descendait de façon continue dans le cas des femmes, dix ans plus tard le niveau se stabilise beaucoup plus vite, puisqu'il se situe aux environs de 4 à $6 \%$ pour les hommes à partir de 28 30 ans et aux environs de $10 \%$ pour les femmes à partir de 30-32 ans. Le taux de chômage des jeunes reste plus élevé que la moyenne, mais à $18 \%(15 \%$ pour les hommes; $21 \%$ pour les femmes), loin des $40 \%$ de 1996 (33\% pour les hommes et $49 \%$ pour les femmes). Le chômage des jeunes reste un problème, mais on peut affirmer qu'il s'agit d'un problème mineur et temporaire, puisque l'intégration dans l'emploi paraît se produire assez rapidement (voir l'article d'Alonso et Martínez dans ce numéro). C'est ainsi que les chômeurs jeunes, qui représentaient $30 \%$ de tous les chômeurs en 1996, sont dix ans plus tard un quart du total, alors que les plus de 40 ans représentent $30 \%$ des chômeurs, contre $22 \%$ dix ans auparavant. Les «jeunes adultes» de 25 à 45 ans sont toujours proches des $50 \%$, même s'il faut noter que, sans les immigrés (qui appartiennent majoritairement à cette tranche d'âge), leur poids dans le chômage aurait légèrement baissé, au détriment des plus âgés.

Quant au niveau d'éducation, les différences subsistent entre ceux qui ont fait des études secondaires complètes (baccalauréat) ou plus, ou bien des études «professionnelles», c'est-à-dire, plus directement liées aux activités productives, et les autres. Pour les premiers, le taux de chômage est inférieur à la moyenne et pour les autres le taux est plus élevé que la moyenne. Malgré cette persistance des différences en termes de taux de chômage, la part du deuxième groupe (ceux qui n'ont que des études secondaires de première étape ou moins), qui était de $64 \%$ en 1996, est descendu à moins de $55 \%$ dix ans plus tard. En somme, bien que les différences persistent, elles tendent à diminuer en partie.

Une question importante en ce qui concerne le chômage est celle de sa persistance dans le temps. Le problème du chômage de longue durée a toujours été un souci pour les responsables de la politique de l'emploi dans tous les pays de l'OCDE, puisque, pour un même niveau de chômage, une longue durée implique des problèmes structurels beaucoup plus graves et difficiles qu'une durée plus courte qui est synonyme d'une répartition plus significative du chômage. Le tableau 2 présente les données relatives au chômage espagnol en 1996 et en 2007. Les résultats sont ceux qu'on pouvait espérer. En effet, au vu des tendances à la réduction des différences du taux de chômage selon les catégories décelées dans les analyses précédentes du tableau 1 - différences persistantes mais certainement amoindries - , la réduction de la durée du chômage ne peut pas être considérée comme surprenante. Si en 1996, 55\% des chômeurs étaient au chômage depuis plus d'un an (chiffre qui s'élevait à $63 \%$ chez les femmes), onze ans plus tard, ils ne sont plus qu'un tiers $(32,7 \%)$ dans ce cas (et seulement $38 \%$ des femmes). 
Tableau 1: Taux de chômage en Espagne en moyennes annuelles (1996 et 2007)

\begin{tabular}{|c|c|c|c|c|c|c|c|c|c|}
\hline & \multicolumn{3}{|c|}{1996} & \multicolumn{3}{|c|}{2007} & \multicolumn{3}{|c|}{2007 (Espagnols) } \\
\hline & Hommes & Femmes & Tous & Hommes & Femmes & Tous & Hommes & Femmes & Tous \\
\hline Ensemble de la population active & 17,2 & 29,6 & 21,9 & 6,4 & 10,9 & 8,3 & 5,8 & 10,2 & 7,6 \\
\hline \multicolumn{10}{|l|}{ Tranches d'âge } \\
\hline $16-24$ & 33,0 & 48,7 & 39,8 & 15,2 & 21,9 & 18,2 & 14,7 & 21,2 & 17,5 \\
\hline $25-40$ & 17,2 & 29,9 & 22,4 & 6,0 & 10,2 & 7,8 & 5,4 & 9,5 & 7,2 \\
\hline $41-54$ & 11,5 & 19,2 & 14,0 & 4,6 & 8,8 & 6,4 & 4,1 & 8,6 & 6,0 \\
\hline $55-64$ & 11,5 & 12,2 & 11,7 & 4,9 & 7,7 & 5,9 & 4,6 & 7,4 & 5,6 \\
\hline 65 et plus & 1,0 & 1,8 & 1,3 & 1,1 & 2,1 & 1,4 & 0,8 & 2,2 & 1,2 \\
\hline \multicolumn{10}{|l|}{ Niveau d'éducation } \\
\hline Analphabètes & 31,6 & 33,9 & 32,7 & 21,3 & 32,1 & 25,3 & 25,4 & 27,0 & 26,1 \\
\hline Études primaires incomplètes & 22,4 & 25,7 & 23,6 & 10,9 & 17,3 & 13,2 & 11,2 & 16,1 & 13,0 \\
\hline Études primaires & 16,9 & 27,0 & 20,0 & 7,8 & 14,1 & 10,0 & 7,5 & 14,1 & 9,8 \\
\hline Études secondaires, première étape & 20,2 & 36,7 & 26,3 & 7,3 & 14,9 & 10,2 & 6,9 & 14,4 & 9,6 \\
\hline FP première étape & 18,0 & 36,7 & 26,2 & 5,6 & 11,3 & 8,2 & 5,3 & 11,4 & 8,1 \\
\hline Baccalauréat & 15,2 & 28,5 & 20,7 & 6,3 & 10,4 & 8,1 & 5,4 & 9,6 & 7,2 \\
\hline FP deuxième étape & 14,9 & 31,9 & 21,2 & 4,3 & 8,4 & 6,0 & 3,9 & 7,8 & 5,5 \\
\hline Études universitaires, premier cycle & 11,5 & 19,9 & 16,2 & 4,3 & 6,1 & 5,3 & 3,8 & 5,6 & 4,8 \\
\hline Études universitaires supérieures & 10,0 & 22,7 & 15,5 & 3,8 & 5,8 & 4,8 & 3,2 & 5,3 & 4,2 \\
\hline \multicolumn{10}{|l|}{ Nationalité et lieu de naissance } \\
\hline $\begin{array}{l}\text { Total Espagnols et Union européenne } \\
\text { dont: }\end{array}$ & 17,1 & 29,6 & 21,9 & 5,8 & 10,2 & 7,6 & & & \\
\hline -Espagnols nés en Espagne & 17,1 & 29,6 & 21,8 & 5,7 & 10,2 & 7,6 & 5,7 & 10,2 & 7,6 \\
\hline -Espagnols nés à l'étranger & 21,5 & 31,6 & 25,9 & 6,2 & 10,3 & 8,1 & 6,2 & 10,3 & 8,1 \\
\hline -Double nationalité & 19,9 & 26,4 & 23,4 & 8,9 & 12,5 & 10,6 & 8,9 & 12,5 & 10,6 \\
\hline $\begin{array}{l}\text { - Citoyens de l'Union européenne } \\
\text { à quinze }\end{array}$ & 10,0 & 21,2 & 14,3 & 8,8 & 11,3 & 9,8 & 8,8 & 11,3 & 9,8 \\
\hline $\begin{array}{l}\text { Total étrangers hors Union eu E, euro- } \\
\text { péenne dont: }\end{array}$ & 25,1 & 23,8 & 24,6 & 8,3 & 12,7 & 10,3 & & & \\
\hline -Europe orientale & & & & 10,6 & 14,2 & 8,4 & & & \\
\hline -Afrique & & & & 13,3 & 32,1 & 16,1 & & & \\
\hline -Amérique latine & & & & 9,6 & 12,5 & 9,7 & & & \\
\hline - Reste & & & & 6,7 & 12,8 & 3,7 & & & \\
\hline
\end{tabular}

Source: microdonnées de l'enquête sur la population active.

Tableau 2 : Répartition des chômeurs selon la durée du chômage (1996 et 2007)

\begin{tabular}{|l|r|r|r|r|r|r|}
\hline & \multicolumn{3}{|c|}{1996} & \multicolumn{3}{c|}{$\mathbf{2 0 0 7}$} \\
\cline { 2 - 7 } & Hommes & Femmes & \multicolumn{1}{|c|}{ Tous } & Hommes & Femmes & \multicolumn{1}{l|}{ Tous } \\
\hline Moins de 6 mois & 33,1 & 22,5 & 27,8 & 56,7 & 45,1 & 50,3 \\
\hline De 6 à 11 mois & 18,8 & 14,6 & 16,7 & 17,3 & 16,8 & 17,0 \\
\hline De 12 à 23 mois & 18,5 & 17,1 & 17,8 & 12,4 & 13,6 & 13,1 \\
\hline 24 mois ou plus & 29,6 & 45,9 & 37,8 & 13,6 & 24,4 & 19,6 \\
\hline Total & 100,0 & 100,0 & 100,0 & 100,0 & 100,0 & 100,0 \\
\hline
\end{tabular}

Source: microdonnées de l'enquête sur la population active.

Une deuxième question sur laquelle il est intéressant d'insister est celle du rôle des ménages en tant qu'amortisseurs du chômage de leurs membres. Comme le montre le tableau 3, alors qu'en 1996 presque $10 \%$ des personnes vivaient dans des ménages où tous les membres actifs se trouvaient en situation de chômage, ce chiffre est de moins de $3 \%$ seulement en 2007. En 1996, un tiers des actifs vivaient dans des ménages affectés par le chômage; onze ans plus tard, le chiffre se réduit à moins de
$15 \%$, soit presque le double du taux de chômage individuel. Cependant, $72 \%$ des chômeurs vivaient en 1996 dans des ménages où d'autres personnes avaient un emploi $(25,2 \%$ sur un total de $34,9 \%$ de chômeurs), chiffre qui augmente jusqu'à $80,6 \%$ en 2007 (7).

(7) Sur le rôle de la famille comme amortisseur des difficultés économiques des chômeurs, voir ToHaRIa (2000). 
En somme, du point de vue des ménages, on peut considérer que le taux de chômage effectif est réellement proche de zéro, bien qu'il demeure 0,8\% de chômeurs $(2 \%$ chez les étrangers hors Union européenne à quinze) qui vivent dans des ménages exclus et de l'emploi et des systèmes de protection sociale (assurance-chômage ou pensions).

Tableau 3 : Répartition de la population selon la situation par rapport à l'activité économique des ménages (1996 et 2006)

\begin{tabular}{|c|c|c|c|c|}
\hline \multirow[b]{2}{*}{$\begin{array}{l}\text { Situation } \\
\text { économique } \\
\text { des ménages }\end{array}$} & \multirow[b]{2}{*}{1996} & \multicolumn{3}{|c|}{2006} \\
\hline & & Tous & Espagnols & $\begin{array}{l}\text { Étrangers } \\
\text { hors Union } \\
\text { européenne } \\
\text { à quinze }\end{array}$ \\
\hline Tous employés & 65,1 & 85,3 & 86,2 & 77,0 \\
\hline $\begin{array}{l}\text { Employés } \\
\text { et chômeurs }\end{array}$ & 25,2 & 11,9 & 11,0 & 19,4 \\
\hline $\begin{array}{l}\text { Tous chômeurs } \\
\text { avec protection } \\
\text { sociale }\end{array}$ & 7,7 & 2,0 & 2,0 & 1,5 \\
\hline $\begin{array}{l}\text { Tous chômeurs } \\
\text { sans protection } \\
\text { sociale }\end{array}$ & 1,9 & 0,9 & 0,8 & 2,0 \\
\hline $\begin{array}{l}\text { Total ménages } \\
\text { «actifs» }\end{array}$ & 100,0 & 100,0 & 100,0 & 100,0 \\
\hline $\begin{array}{l}\text { \% de ménages } \\
\text { avec tous } \\
\text { les membres } \\
\text { inactifs }\end{array}$ & 13,5 & 12,8 & 13,9 & 2,3 \\
\hline
\end{tabular}

Source: microdonnées de l'enquête sur la population active.

\section{'évolution du marché}

\section{du travail espagnol dans une perspective comparée}

Pour mettre en perspective l'évolution du marché du travail espagnol décrite dans la section précédente, il est intéressant de la comparer avec l'expérience de certains autres pays européens, qui représentent les différents modèles d'État-providence existant en Europe: la Suède, le Royaume-Uni et la France. Le graphique 4 présente la comparaison en ce qui concerne les taux d'emploi; le graphique inclut aussi les chiffres pour l'ensemble de l'Union européenne à quinze. Le rattrapage qui se produit en ce qui concerne l'Espagne par rapport aux autres pays et notamment par rapport à la France est très visible dans le graphique. En 2005, l'Espagne surpasse le taux d'emploi français et se rapproche très vite de la moyenne européenne. Évidemment, les chiffres espagnols et français demeurent assez loin des niveaux suédois et britanniques, mais il est clair que l'Espagne est le seul pays à connaître une augmentation de son taux de façon continue tout au long de la période observée et que le ralentissement qui s'observe après 2001 dans tous les autres pays de référence ne se produit pas en Espagne.
Pour compléter la comparaison internationale, le graphique 5 présente l'évolution du taux de chômage pour la même période et les mêmes pays considérés dans le graphique précédent. Comme dans le graphique précédent, la convergence de l'Espagne vers les autres pays est évidente, jusqu'au point qu'en 2006 le taux de chômage espagnol est inférieur à celui de la France.

En somme, l'Espagne qui a été pendant presque vingt ans le pays de l'Europe qui souffrait du taux de chômage le plus élevé se trouve actuellement beaucoup plus près de la moyenne européenne. S'agitil d'un processus sans retour, d'un changement structurel qui rapproche l'Espagne du reste des pays européens qui lui sont proches? Les données présentées dans les graphiques 4 et 5 le suggèrent, et les prévisions qui existaient jusqu'en 2007 n'indiquaient pas qu'il pourrait y avoir des problèmes tendant à interrompre les tendances observées. D'après ces prévisions, semblait-il, l'Espagne aurait été capable de surmonter l'amplitude qui avait caractérisé les évolutions passées de l'emploi et du chômage et, en particulier, les crises des années 1977-1984 et 1992-1994, jugées de nature structurelle. Les données existantes au début 2008, cependant, suggèrent la possibilité d'une nouvelle crise, liée aux phénomènes étudiés dans ce numéro: modèle de développement lié a une baisse de productivité (voir article de Cebrián), emploi précaire (voir article de Miguélez et Prieto), etc. Dans la section suivante, trois problèmes structurels de l'économie espagnole sont mentionnés à titre d'exemple.

\section{Des différences régionales, une composition sectorielle de l'emploi et un niveau d'éducation problématiques}

Reste-t-il, dans la situation qui vient d'être présentée, des lacunes qui pourraient poser des problèmes au marché du travail espagnol dans le futur? Sans prétendre à l'exhaustivité, trois peuvent être brièvement mentionnées. Dans le reste de ce numéro, d'autres questions complémentaires seront soulevées.

En premier lieu, les différences persistent, bien que certainement amoindries, entre les diverses catégories de chômeurs. L'une des différences les plus persistantes est celle qui existe entre les régions espagnoles. Comme le montre le graphique 6 , les taux de chômage ont baissé très notablement dans toutes les régions, de telle sorte qu'en moyenne ils représentent en $200640 \%$ de ceux de 1996. Mais la corrélation entre les taux dans les deux moments d'observation est très élevée, ce qui indique que les différences subsistent. Même si, en moyenne, le taux de chômage en 2007 est de 8,5\%, dans certaines 
Graphique 4 : L'évolution du taux d'emploi en Espagne, France, Royaume-Uni, Suède et Union européenne à quinze (1994-2006)

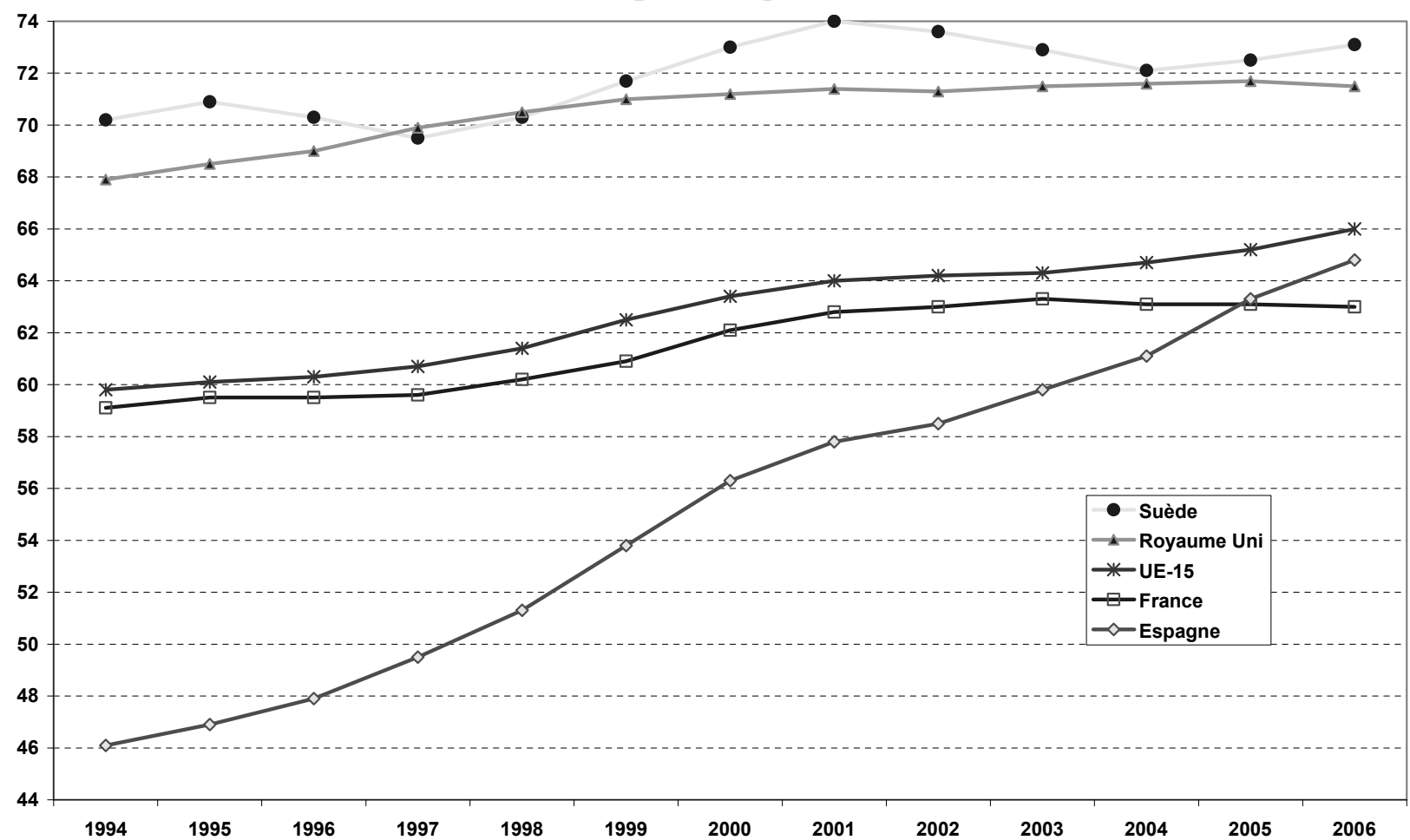

Source: Eurostat, Commission européenne, site (http ://ec.europa.eu/eurostat).

Note de lecture: le graphique montre les données provenant de l'enquête européenne sur les forces de travail qui couvre l'ensemble de la population vivant dans des ménages privés. Le taux d'emploi est obtenu en divisant le nombre de personnes occupées âgées de 15 à 64 ans par la population totale de la même tranche d'âge. Les données nationales sont ajustées par l'Eurostat.

\section{Graphique 5: L'évolution du taux de chômage en Espagne, France, Royaume-Uni, Suède et Union européenne à quinze (1994-2006)}

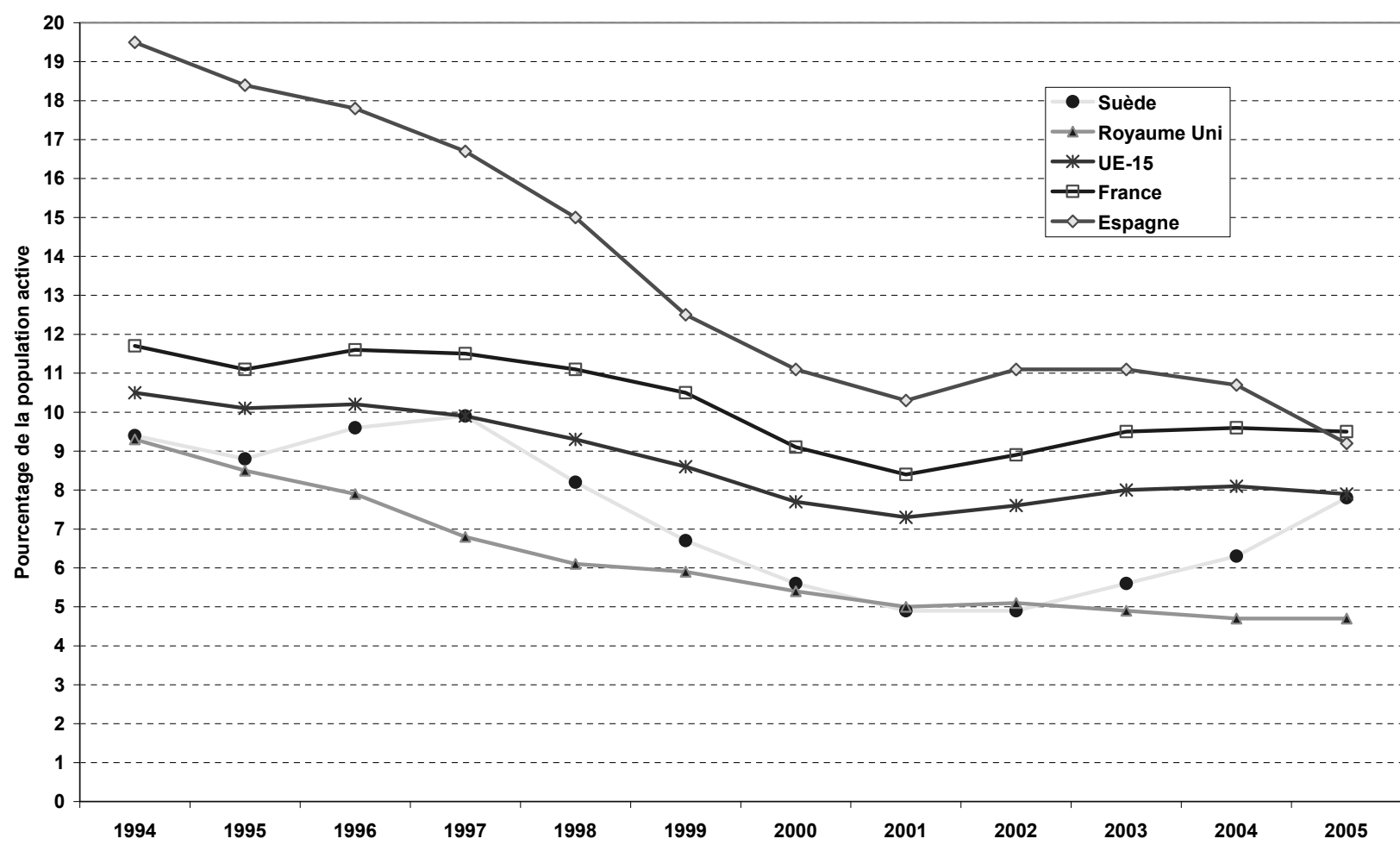

Source: Eurostat, Commission européenne, site (http://ec.europa.eu/eurostat).

Note de lecture: le graphique montre les données provenant de l'enquête européenne sur les forces de travail qui couvre l'ensemble de la population vivant dans des ménages privés. Le taux de chômage est le pourcentage de chômeurs de 15 à 74 ans par rapport à l'ensemble des forces de travail. Les données nationales sont ajustées par Eurostat. 


\section{Graphique 6: La persistance des différences régionales taux de chômage en 1996 et en 2007, selon le sexe et la région de résidence}

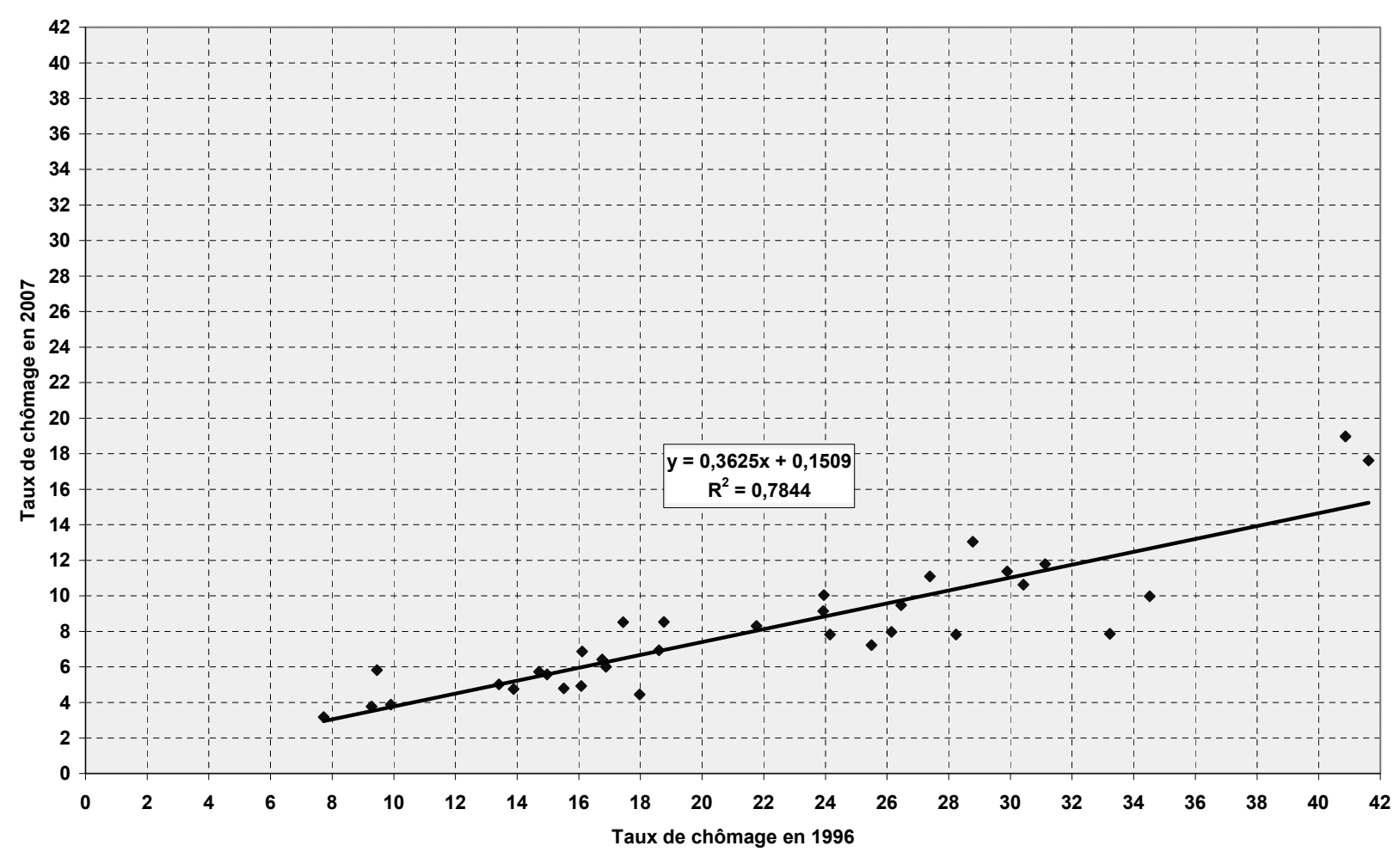

Source: microdonnées de l'enquête sur la population active.

Note de lecture: le graphique montre la relation existant entre les taux de chômage par région et sexe (trente-quatre observations) observés en 1996 et en 2007. La diagonale principale du graphique (qui n'est pas explicite dans le graphique) montrerait une situation de persistance totale des niveaux. La ligne de régression représentée est plus plate et au-dessous de la diagonale, ce qui indique une réduction générale des taux de chômage, mais aussi une persistance des différences (coefficient de corrélation élevé) bien qu'atténuées (pente de la ligne inférieure à l'unité).

régions, notamment celles du Sud de l'Espagne, le taux est supérieur à $13 \%$, et atteint $18-19 \%$ chez les femmes. Le développement économique n'a pas été capable, par conséquent, de produire un rapprochement des niveaux de vie des régions les plus défavorisées avec celui des plus avancées. Il est remarquable, quand même, que ces différences ne se soient pas creusées, mais leur existence indique que les politiques de compensation interrégionale devront continuer, si l'on vise la cohésion territoriale, et cela aussi bien avec l'aide des régions espagnoles les plus riches qu'avec les fonds structurels de l'Union européenne, destinés à diminuer dans les années à venir. Il est peu probable, néanmoins, qu'une éventuelle crise économique forte aggrave les différences régionales mentionnées.

En deuxième lieu, bien que cet article ait analysé principalement la question du chômage, la forte croissance de l'emploi pose la question des secteurs dans lesquels l'Espagne s'est spécialisée. L'article de Cebrián répond à cette question de façon détaillée. Ce qu'il est intéressant de voir ici, c'est la comparaison entre la structure de l'emploi qui existe en Espagne et celle de ses partenaires européens. Le tableau 4 présente des données à partir desquelles on peut extraire cinq conclusions importantes:
- le poids de l'agriculture, bien que très inférieur à ce qu'il était vingt ou trente ans auparavant (en 1977 le chiffre était de 23\%), demeure relativement élevé;

- la construction a un poids extraordinaire dans l'économie espagnole; on peut affirmer, sans peur de se tromper, que le bâtiment a été le secteur moteur de l'économie espagnole; les périls que ce surdéveloppement entraîne sont déjà malheureusement perceptibles et deviennent une réalité préoccupante au début 2008 ;

- le tourisme est un des secteurs les plus forts de l'économie espagnole, comme le démontre le poids plus visible qu'ailleurs du secteur des hôtels et restaurants; la situation internationale a été favorable au secteur touristique espagnol, mais il s'agit d'une industrie assez bien développée qui, malgré les risques inhérents du point de vue de l'aménagement du territoire et de l'environnement, paraît assez solide face au futur;

- les services collectifs, notamment éducation et santé, demeurent sous-développés par rapport à la moyenne des pays plus avancés; la décentralisation de ces services essentiels limite la capacité du gouvernement de l'État à développer ce secteur qui manifeste d'ores et déjà des besoins de plus en plus forts à cause du vieillissement de la population 
Tableau 4 : Composition de l'emploi par branches d'activité économique (2007)

\begin{tabular}{|l|r|r|r|r|r|r|r|r|}
\hline & Allemagne & France & Italie & Suède & Royaume-Uni & Espagne & $\begin{array}{c}\text { Union européenne } \\
\text { à quinze }\end{array}$ & $\begin{array}{c}\text { Union européenne } \\
\text { à vingt-cinq }\end{array}$ \\
\hline Agriculture & 2,2 & 3,4 & 4,0 & 2,2 & 1,4 & 4,5 & 3,5 & 4,5 \\
\hline Industrie & 23,2 & 16,4 & 21,7 & 15,2 & 13,9 & 16,0 & 18,1 & 19,1 \\
\hline Construction & 6,6 & 6,9 & 8,4 & 6,4 & 8,2 & 13,3 & 8,2 & 8,2 \\
\hline Commerce & 14,0 & 13,8 & 15,2 & 12,2 & 14,4 & 15,4 & 14,5 & 14,5 \\
\hline Hôtels et restaurants & 3,8 & 3,4 & 5,0 & 3,1 & 4,4 & 7,1 & 4,6 & 4,3 \\
\hline $\begin{array}{l}\text { Transports et } \\
\text { communications }\end{array}$ & 5,6 & 6,3 & 5,4 & 6,2 & 6,7 & 5,8 & 6,0 & 6,1 \\
\hline Activités financières & 3,4 & 3,2 & 2,9 & 2,0 & 4,3 & 2,5 & 3,2 & 3,1 \\
\hline $\begin{array}{l}\text { Immobilier, location et } \\
\text { services aux entreprises }\end{array}$ & 10,3 & 10,4 & 10,9 & 14,6 & 12,0 & 9,9 & 10,6 & 10,0 \\
\hline Administration publique & 7,6 & 10,1 & 6,1 & 5,7 & 7,0 & 6,1 & 7,3 & 7,2 \\
\hline Éducation & 5,9 & 6,8 & 6,9 & 10,8 & 9,1 & 5,5 & 6,9 & 7,0 \\
\hline Santé et action sociale & 11,4 & 12,3 & 6,8 & 15,9 & 12,1 & 6,0 & 10,5 & 9,8 \\
\hline Autres services collectifs & 5,4 & 4,5 & 5,0 & 5,3 & 5,7 & 4,2 & 4,9 & 4,7 \\
\hline $\begin{array}{l}\text { Ménages employeursde } \\
\text { personnel domestique }\end{array}$ & 0,5 & 2,3 & 1,5 & 0,0 & 0,5 & 3,8 & 1,3 & 1,2 \\
\hline Total & 100,0 & 100,0 & 100,0 & 100,0 & 100,0 & 100,0 & 100,0 & 100,0 \\
\hline
\end{tabular}

Source: base de données d'Eurostat.

espagnole lié à la baisse de la fécondité; on l'a vu lors de la mise en place de la loi sur la protection des personnes dépendantes, qu'il appartient aux régions de développer et qu'elles appliquent avec des résultats irréguliers et des frictions politiques ou concernant le financement des dépenses impliquées (voir l'article de Rodríguez Cabrero);

- pour finir, et de manière partiellement liée au point précédent, le poids du service domestique est beaucoup plus élevé qu'ailleurs en Europe, du fait notamment du recours à ce secteur pour s'occuper des personnes âgées. L'article de Prieto et Miguélez développe les problèmes que les emplois précaires peuvent poser à l'économie espagnole dans le futur.

Pour conclure, un problème important subsiste pour l'avenir du marché du travail espagnol. Il s'agit du niveau d'éducation de la population espagnole, et en particulier des jeunes. Du point de vue de l'évolution de l'emploi et du chômage, cela ne semblerait pas poser de grands problèmes à l'économie espagnole, particulièrement si le modèle espagnol de croissance se prolonge, ce qui implique des bas niveaux de productivité, comme le démontre Immaculada Cebrián dans son article. Mais si l'Espagne veut arriver à jouer un rôle plus décisif dans l'ordre économique européen, il est probable que le capital humain prendra une place importante. La crise qui semble se faire jour en cette fin de la première décennie du $\mathrm{XXI}^{\mathrm{e}}$ siècle renforce cette idée. Il est donc nécessaire de voir quelle place occupe actuellement l'Espagne à cet égard.

Le tableau 5 montre trois indicateurs du développement éducatif de divers pays européens, calculés par Eurostat à partir de données comparables de l'enquête communautaire des forces de travail.
Les jeunes quittant prématurément l'école sont les jeunes de 18 à 24 ans qui répondent aux deux caractéristiques suivantes: le plus haut niveau d'enseignement ou de formation atteint est le niveau de base (CITE 0, 1, 2 ou 3c court) et les répondants ont déclaré n'avoir suivi aucun enseignement ni aucune formation au cours des quatre semaines précédant l'enquête. Ce premier indicateur atteint une valeur pour l'Espagne double de celle des pays européens plus développés. Bien qu'il soit possible que ces jeunes retournent ultérieurement à l'école, il est assez probable qu'ils n'arriveront jamais à atteindre le niveau secondaire supérieur.

Le deuxième indicateur est défini comme le pourcentage des jeunes de 20 à 24 ans ayant au moins atteint un niveau d'enseignement ou de formation secondaire supérieur(8) (numérateur). L'Espagne se trouve encore une fois à mi-chemin entre le Portugal et les pays plus développés, bien que les différences semblent, dans ce cas, moins troublantes. Néanmoins, comme dans le cas du premier indicateur, il faut signaler que les tendances historiques ne montrent pas une amélioration des chiffres.

Enfin, le troisième indicateur donne le pourcentage de la population adulte (25-64 ans) qui a achevé le deuxième cycle des études secondaires. Il vise à mesurer la part de la population susceptible de posséder les qualifications minimales nécessaires pour prendre une part active dans la vie économique et sociale(9). Les différences entre l'Espagne et les

(8) Soit un niveau CITE 3a, 3b ou 3c long minimum.

(9) Eurostat souligne qu'il «convient de noter que le deuxième cycle des études secondaires peut être effectué dans les pays européens après des études de longueurs variables suivant les différents systèmes éducatifs nationaux ». 
pays européens plus développés sont également assez notables en ce qui concerne cet indicateur, bien que le chiffre espagnol suive une tendance croissante, explicable par le niveau plus élevé des jeunes par rapport à leurs aînés.

En résumé, le niveau d'éducation des jeunes espagnols semble sensiblement en retard par rapport à celui des jeunes des autres pays européens. Comme on l'a vu dans le tableau 4, il semble que l'éducation soit un secteur sous-développé, dont les résultats ne sont pas très satisfaisants en Espagne. Si l'Espagne veut éviter les crises périodiques d'un modèle de développement excessivement basé sur le bâtiment et les emplois précaires, elle devra faire un effort supplémentaire pour accroître le niveau d'éducation de ses jeunes.

Tableau 5 : Indicateurs du niveau d'éducation de la population en Europe (2007)

\begin{tabular}{|l|r|c|c|}
\hline & $\begin{array}{c}\text { Jeunes } \\
\text { quittant } \\
\text { préma- } \\
\text { turément } \\
\text { l'école }\end{array}$ & $\begin{array}{c}|c| \\
\text { Population ayant au } \\
\text { moins terminé } \\
\text { le cycle secondaire } \\
\text { supérieur }\end{array}$ \\
\cline { 2 - 4 } & $18-24$ ans & $20-24$ ans & $25-64$ ans \\
\hline Allemagne & 12,7 & 72,5 & 84,4 \\
\hline Espagne & 31,0 & 61,1 & 50,4 \\
\hline France & 12,7 & 82,4 & 68,7 \\
\hline Italia & 19,3 & 76,3 & 52,3 \\
\hline Pays-Bas & 12,0 & 76,2 & 73,2 \\
\hline Portugal & 36,3 & 53,4 & 27,5 \\
\hline Royaume-Uni & $13,0^{(*)}$ & 78,1 & 73,3 \\
\hline Suède & $12,0^{(*)}$ & 87,2 & 84,6 \\
\hline $\begin{array}{l}\text { Union européenne à } \\
\text { vingt-cinq }\end{array}$ & 14,5 & 78,1 & 70,5 \\
\hline
\end{tabular}

(*) 2006 .

Source: base de données Eurostat$$
\text { * * }
$$

Tout au long de cet article, nous avons présenté les données fondamentales du marché du travail espagnol afin de fournir les éléments de base pour les analyses plus détaillées des divers aspects qui constituent le reste de ce numéro spécial. Nous récapitulons ici les principaux résultats obtenus dans l'article:

1) Entre 1976 et 2007 , le marché du travail espagnol a subi deux fortes crises et deux fortes périodes d'expansion; au total, la situation à la fin de la période examinée montre un accroissement plus que visible des niveaux d'emploi et une réduction des niveaux de chômage qui, malgré tout, dépasse encore les $8 \%$ de la population active et risque de s'aggraver au cours des prochaines années.

2) Cette évolution a été fortement différente pour les hommes et pour les femmes; pour les premiers, la situation a suivi un mouvement qui fait penser à un $\mathrm{W}$ dans le cas de l'emploi et à un $\mathrm{M}$ dans le cas du chômage, et l'augmentation de l'emploi depuis 2000 n'a été possible que grâce aux fortes entrées de travailleurs provenant d'autres pays, notamment le Maroc et l'Amérique latine (mais aussi, plus récemment, la Roumanie); dans le cas des femmes, l'activité s'est accrue de façon continue depuis les premières années 1980 .

3) Le résultat de cette évolution est que le marché du travail espagnol se trouvait, en 2007, dans une situation inconnue depuis trente ans, bien que le taux de chômage des jeunes, des femmes, des immigrés demeure plus élevé que le chômage de longue durée, qui était très significatif dans les années 1990, soit beaucoup moins important et que la situation économique des chômeurs, mesurée par la situation des ménages dans lesquels ils vivent, se soit beaucoup améliorée : en 2007,3\% des chômeurs vivent dans des ménages où tous les membres actifs sont chômeurs, le pourcentage étant proche des $10 \%$ dix ans avant.

4) Trois importantes lacunes ont été soulignées, cependant, dans ce panorama relativement optimiste. Premièrement, d'importantes différences régionales subsistent, bien qu'il soit vrai que toutes les régions ont bénéficié de la croissance économique; mais avec des taux de chômage féminins encore proches de $20 \%$, les régions du Sud de l'Espagne demeurent éloignées des hauts niveaux d'emploi des régions plus développées. Deuxièmement, la structure économique de l'emploi repose de façon plus prononcée qu'ailleurs en Europe sur la construction, le tourisme (hôtels et restaurants) et les ménages employeurs de personnel domestique, des secteurs à basse productivité, et moins sur les services plus avancés (services aux entreprises et activités financières) et sur les services sociaux (notamment, éducation et santé). Finalement, le grand bond en avant qu'a connu le niveau d'éducation de la population espagnole au cours des trente dernières années s'avère insuffisant pour arriver à des niveaux comparables à ceux des partenaires européens: le pourcentage de jeunes qui ne dépasse pas le niveau secondaire (premier cycle) reste trop élevé et les tendances des dernières années n'indiquent pas un mouvement d'amélioration; le fait que la plupart de ces jeunes «sous-éduqués » trouvent un emploi n'est qu'une indication des types d'emplois qui ont été créés en Espagne; en même temps, cela pose des questions sur les possibilités d'adaptation du modèle actuel espagnol de croissance et sur sa capacité à évoluer vers un autre modèle à plus grande valeur ajoutée, qui permettrait des gains de productivité plus prononcés et à long terme de trouver un chemin de croissance plus stable et plus sûr.

Les articles contenus dans ce volume approfondissent les questions qui viennent d'être mentionnées et qui deviennent particulièrement importantes au vu des prévisions et des données les plus récentes qui situent déjà l'Espagne à la tête du ralentissement économique européen. 


\section{Bibliographie}

Anxo D., Cebrián I., Fagan C., Moreno G. (2007), "Patterns of Labour Market Integration in Europe: A Life Course Perspective on Time Policies", Socio-Economic Review, Vol. 5, avril, pp. 233-260.

Cebrián I., Moreno G. (2007), "La situación de las mujeres en el mercado de trabajo español. Desajustes y retos", Economía industrial, no 367 , pp. 121-137.

Cebrián I., Moreno G., Toharia L. (2007), “Transiciones de hogares y transiciones de oferta de trabajo", in El mercado de trabajo europeo en el proceso de convergencia económica y social: un análisis basado en el panel de hogares de la Unión Europea (PHOGUE), 1994-2001, Fundación CaixaGalicia, pp. 60-120.

COMmission EuRopéEnNe (2007), L'emploi en Europe 2007, Bruxelles, Eurostat,

Garrido L. (1993), Las dos biografías de la mujer en España, Madrid, Institut de la femme.

Garrido L., Toharia L. (2004), "What does it take to be (counted as) unemployed? The case of Spain", Labour economics, Vol. 11, no 4, pp. 507-523.

INE (Instituto Nacional de Estadística) (plusieurs années), Encuesta de Población Activa, microdonnées.

Toharia L. (1998), «La politique de l'emploi en Espagne», in Barbier J.-C. et Gautié J., (sous la direction de), Les politiques de l'emploi en Europe et aux ÉtatsUnis, Paris, PUF, pp. 93-112.

TOHARIA L. (2000), "El paro en España: ¿puede ser tan alto?", Revista gallega de empleo, $\mathrm{n}^{\circ} 0$, février, pp. 75104.

Toharia L., Malo M.A. (2000), "The Spanish experiment: the pros and cons of the flexibilty at the margin", in Esping-Andersen, G. et Regini M., (eds), Why Deregulate Labour Markets? Oxford University Press, pp. 307-335.

TOHARIA L. (2005), "Empleo y paro en España: situación y perspectivas”, CLM.economía, no 4, 2005.

\section{Bibliographie complémentaire}

Les lecteurs intéressés peuvent trouver des informations complémentaires sur la situation générale, l'évolution et les perspectives du marché du travail espagnol dans les textes suivants (entre autres):

COMISIÓn de EXPERTOS PARA El DiÁlogo SOCIAL, Más y mejor empleo en un nuevo escenario socioeconómico - Por una flexibilidad y seguridad laborales efectivas, Madrid, Ministerio de Trabajo y Asuntos Sociales, 2005. Étude de base pour le processus de dialogue social aboutissant à la réforme du marché du travail de juin 2006.

Consejo Económico y Social, Economía, trabajo y sociedad - Memoria sobre la situación socioeconómica y laboral de España, Madrid, CES. Rapport annuel du Conseil économique et social sur la situation socioéconomique de l'Espagne.

ObSERVATORIO SOCIAL DE ESPAÑA, groupe dirigé par le professeur Vicenç Navarro, site web avec une base de données sur la situation sociale en Espagne et des références aux volumes annuels publiés par le groupe. 